\title{
Can focus groups be a tool for change? Introducing health equity to environmental public health practice
}

\author{
Karen Rideout ${ }^{*, \dagger}$, Dianne Oickle ${ }^{*}$ and Connie Clement \\ *BC Centre for Disease Control, Vancouver, BC. \\ †National Collaborating Centre for Environmental Health, Vancouver, BC. \\ ${ }^{*}$ National Collaborating Centre for Determinants of Health, Antigonish, NS.
}

\begin{abstract}
This qualitative investigation was undertaken to explore the value of focus group participation to introduce new concepts into practice within public health. Seven public health inspectors who participated in an earlier focus group study responded to follow-up questions designed to assess whether their participation in the original focus group sessions lead to changes in their thinking or practice. Findings suggest that focus group participation can provide an opportunity to start conversations about new concepts, highlight ways to put thoughts into action, validate how current practice supports broader goals, and identify gaps and next steps. Although an important tool for change, systematic change requires additional support at the organizational level to achieve full implementation. Further research into the use of focus groups as a tool for reflective practice is recommended.
\end{abstract}

Key words: environmental health, focus groups, health equity, knowledge translation, public health practice, social determinants of health.

We conducted focus groups with public health inspectors (PHIs) in 2014 as part of a pilot study to assess how health equity and the social determinants of health (SDH) intersect with their practice. That study, published elsewhere, focused on where social barriers affect compliance, how practitioners respond, and where there are barriers that limit practitioner response (Rideout and National Collaborating Centre for Determinants of Health, 2015; Rideout and Oickle, 2016). Informal feedback from participants during the focus groups suggested that taking part in the sessions had value for them in several ways, including the opportunity to discuss issues they may not have otherwise considered. Because SDH and health equity are relatively new areas of practice for PHIs, we considered how focus group participation may have influenced participants' thinking and practice, as well as whether focus groups might be useful for enabling changes in public health practice related to health equity.

In addition to their use in exploratory research (The Health Communication Unit, 2002), focus groups have been indicated as a tool for implementing process changes within the health sector. In particular, focus groups can increase awareness and knowledge related to what needs to change, how and why to make the change, and they can identify barriers and facilitators to change, all of which may reduce resistance to change (National Institute for Health and Clinical Excellence, 2007; Stevens et al., 2007).

Corresponding author: Karen Rideout (email: karen.rideout@bccdc.ca)
In this exploratory study, we build on the findings of the pilot study to examine the potential of focus groups as a tool to introduce new concepts, link theoretical concepts with the realities of frontline practitioners, and encourage public health professionals to change their practice accordingly.

\section{Methods}

The pilot study involved two focus groups with PHIs in British Columbia and Nova Scotia. Each one- to two-hour focus group session was moderated using a semi-structured interview guide, was conducted in person, and included five to six participants with a mix of experience (Rideout and National Collaborating Centre for Determinants of Health, 2015). The consent form for the study, which was approved by the Fraser Health Research Ethics Board and the University of British Columbia Behavioural Research Ethics Board, indicated that participants may be contacted for further follow-up. In July 2015, we sent an email to all 11 original focus group participants to request their participation in a short follow-up questionnaire about their experience of participating in the focus group. We embedded six questions in the email invitation, and participants were invited to respond via email or call the researchers to set up a telephone interview.

The questionnaire (Figure 1) asked participants several open-ended questions about their recollection of participating in the March 2014 focus groups and of reviewing the April 2015 study report (Rideout and National Collaborating Centre 
1. Do you remember participating in the focus group?

2. Have you discussed your experience of participat ing in the focus group with colleagues?

(e.g., which colleagues, topic of discussion, etc.)

3. Has the experience of participating in this focus group changed your thinking or your practice in any way? If so, how?

4. Have you approached colleagues or management with any of the specific issues that came to mind through your participation in this project?

5. Have you read the report?

6. Have you shared or discussed the report with colleagues?

(e.g., which colleagues, topic of discussion, etc.)

Figure 1. Follow-up questions posed to focus group participants to assess how participation may have influenced thinking or practice.

for Determinants of Health, 2015). Other questions were designed to look more deeply at whether their participation in the focus group led to conversations with colleagues, changes in thinking or practice, or pursuit of integrating SDH and health equity into environmental health practice at an organizational level.

Questionnaire responses were entered into an Excel spreadsheet and both authors conducted a qualitative thematic analysis of the responses to each question. We first reviewed the responses for key concepts, manually coded them in Excel, and then grouped the codes into the themes that form the findings presented in the next section.

\section{Results}

Seven of the original 11 focus group participants responded to the questionnaire via email (one person was no longer in her position; three were non-responders). All respondents remembered participating in the focus groups and most read the report. Although none purposely shared the report with colleagues or management, it had been circulated in two workplaces and two respondents planned to share it following this questionnaire. Responses suggest that focus group participation presents opportunities at four "stops" along a path where participants can explore concepts discussed in the focus groups (health equity, SDH) and how they relate to environmental public health practice (Figure 2).

\section{Start the conversation: discussion points to explore with colleagues or managers}

All respondents indicated that they discussed the focus group with their colleagues. They talked about opinions and perspectives of the other PHIs in the focus groups, and started to explore solutions. They consistently pointed out that change would require involvement beyond the frontline practitioner level:

\footnotetext{
We agreed that there are many different issues; however, we never really came up with a solution as we felt they were more management (or health authority) wide solutions and not just our office or individual solutions.
}

Some participants also reported realizing that their role could be enriched beyond what they currently focus on:

It made me realize that we could be supported by more resources as EHOs [environmental health officers] in the field.

\section{Shift from thought to action: considerations for how to proceed}

Five of the respondents indicated that the experience of participating in this focus group changed their thinking or practice in some way. They reported subtle shifts in awareness after discussing issues in the focus group, suggesting that the experience of participating in a focus group can change perspective:

It has, [like] all experiences in life, changed the way we think, no matter whether we consciously think about it or not.

\section{Start the \\ conversation}

3. Validate existing

efforts

\author{
2. Shift from \\ thought to action
}

4. Identify next

steps

Figure 2. Continuum of where focus groups can help introduce new concepts and lead to shifts in practice. 
Exposure to conversations about issues such as the SDH and health equity can contribute to a change in thinking that, when in the field, may contribute to a change in action.

While only two participants indicated that participating in the focus groups did not change thinking or practice, their reasons revealed some deeper organizational issues that may be perceived as barriers:

Not really, as some of the concerns/issues that arise still require programs to be in place to assist the vulnerable/lower income.

In addition to the feeling that their thinking wasn't changed because of the lack of programs, there was an indication that practitioners can feel powerless in the wake of broader mandates for public health action that supersede what they might do as individuals:

In some ways it has (changed my thinking) but in others we unfortunately cannot change how things are done due to budget and mandate. I have tried to reach out to lower income families by providing information through such places as libraries. Some issues like food safety in the home are done more on an overall health authority situation and I think they need to change how those programs as delivered as well, I cannot change those.

\section{Validate existing efforts: sharing ideas for changing practice}

Several participants indicated that being part of the group discussion "helped to validate" some of what focus group participants had already been feeling. The experience showed them how to put ideas into practice, reinforced that they are contributing to broader public health goals, or let them know that they are not alone in the challenges they see through their work. What they took to be just "doing their job" was in fact applying an equity lens, regardless of terminology used. The focus group seemed to provide validation of those efforts:

There was comfort in knowing we all had similar experiences when in the field.

Both the group discussion and reading the paper helped me to see that some of the challenges I face in the job are somewhat similar to others in my profession.

One participant stated that the focus group discussions supported the broader perspective they had of their overall work and the organizational structure they are working in:

It reinforced to me the complexity of our field and our Health Authority and the danger in a blanket application of policies and guidelines across the health authority.

\section{Identify next steps: highlighting gaps and support for role definition}

Only two participants approached their colleagues or managers about specific issues that came to mind during the focus groups. However, their experience highlighted organizational barriers that prevent them going beyond their traditional roles and considering different ways of doing frontline work.
Management was not open to change at this time due to many factors.

One participant indicated that the workplace environment was supportive of exploring issues on an organizational level through workforce development:

Late last year I participated in a workforce development strategy working group, and some of my colleagues in that group brought up some of the same challenges and barriers for workforce development. This group consisted of all levels of staff (management to admin support) and in all areas related to population health (public health nurses, dietitians, speech therapists, etc.) and health protection (EHOs, LOs [licensing officers], etc.)

\section{Discussion: moving from talk to action}

This small study suggests that conversations such as focus groups can be useful to support change in public health practice. The four themes highlighted here - start the conversation, shift from thought to action, validate existing efforts, and identify next steps - show how focus group participation can serve as a form of critical reflective practice. Studying one's own experiences to improve the way one works may inform public health practice, no matter the purpose or topic of the original focus group. Reflective capacity is seen as an essential competency for professional practice (Mann et al., 2007). For public health nurses, participation in reflection and dialogue with peers and supervisors allows them to debrief experiences, develop skills in clinical reasoning, and identify best practices (Tanner, 2006). The debriefing that occurs through reflective practice may help staff manage stress and other emotions that arise from involvement in demanding situations during their daily practice (Beam et al., 2010), thereby improving their capacity to address needs as they arise.

These focus groups with environmental health staff helped introduce the concepts of health equity and SDH, illustrated their relevance to practice, and showed how the approach of individual practitioners can fit within broader public health goals. While this is essential for buy-in and implementation of new ways of working, it was clear from this follow-up study that focus group participation alone is not sufficient to enact major changes in how people work. Managerial and organizational changes are needed to enable action at the front lines. Leadership and expertise in organizational change are thought to be important factors in facilitating the shift of priorities or allocation of resources in order to tackle SDH and health equity (National Collaborating Centre for Determinants of Health, 2015). Contextual factors such as institutional policies, organizational hierarchy, decision-making processes, and leadership also influence the successful integration of equity work (Kezar et al., 2008). However, participants may gain knowledge and perspective that facilitates acceptance of new policy directives, uptake of training opportunities, or receptivity to changing goals, visions, policies, or procedures. It may also inspire "champions" who will drive innovation within an organization (Howell et al., 2005). The four-stop pathway identified here can result from focus groups no matter what the instigating purpose might have been.

There are several limitations to this study. It is based on a sub-sample of participants from a small pilot study. 
As a qualitative investigation with participants from a single professional discipline, no assumptions can be made regarding the generalizability of the findings to this or other disciplines. Because participants were frontline staff, these findings do not reflect the perspectives of practitioners at other levels, such as management.

These findings identified a need for organizational or managerial support to facilitate practice change after concepts have been introduced during an event such as a focus group. Management-sanctioned focus groups may create an expectation that change will follow. It is therefore important to be clear about the purpose when a focus group is carried out, so that participants know whether the discussion is simply to explore an issue or is the start of a planned change process. Further investigation into how participation in focus groups leads to practice change as a result of the opportunity to explore concepts would support the use of reflective practice to develop skills and competencies.

\section{Acknowledgements}

The authors wish to acknowledge the focus group participants who responded to our follow-up questions about their participation in the original study and Tom Kosatsky for his contributions to conceptualizing this manuscript.

\section{References}

Beam, R.J., O’Brien, R.A., and Neal, M. 2010. Reflective practice enhances public health nurse implementation of nurse-family partnership. Public Health Nurs. 27(2): 131-139. doi: 10.1111/ j.1525-1446.2010.00836.x.

Howell, J.M., Shea, C.M., and Higgins, C.A. 2005. Champions of product innovations: Defining, developing, and validating a measure of champion behavior. J. Bus. Venturing. 20(5): 641661. doi: 10.1016/j.jbusvent.2004.06.001.
Kezar, A., Glenn, W.J., Lester, J., and Nakamoto, J. 2008. Examining organizational contextual features that affect implementation of equity initiatives. J. High Educ. 79(2): 125-159. doi: 10.1353/ jhe.2008.0006.

Mann, K., Gordon, J., and MacLeod, A. 2007. Reflection and reflective practice in health professions education: A systematic review. Adv. Health Sci. Educ. 14(4): 595-621. doi: 10.1007/ s10459-007-9090-2.

National Collaborating Centre for Determinants of Health. 2015. Learning to work differently: Implementing Ontario's social determinants of health public health nurse initiative. Antigonish, NS. Available at: http://nccdh.ca/resources/entry/learning-to-workdifferently-implementing-ontarios-sdoh-public-health-nurse

National Institute for Health and Clinical Excellence. 2007. How to change practice. Understand, identify and overcome barriers to change. London: National Institute for Health and Clinical Excellence.

Rideout, K., and National Collaborating Centre for Determinants of Health. 2015. Equity in environmental health practice: Findings of a pilot study. Vancouver, BC and Antigonish, NS. Available at: http:// www.ncceh.ca/documents/evidence-review/equity-environmentalhealth-practice-findings-pilot-study

Rideout, K., and Oickle, D. 2016. Integrating equity into environmental health practice: Findings of a pilot study. Environ. Health Rev. 59(1): 35-39. doi: 10.5864/d2016-009.

Stevens, B., Lee, S.K., Law, M.P., Yamada, J., Canadian Neonatal Network, \& EPIC Study Group. 2007. A qualitative examination of changing practice in Canadian neonatal intensive care units. J. Eval. Clin. Pract. 13(2): 287-294. doi:10.1111/j.1365-2753.2006.00697.x.

Tanner, C.A. 2006. Thinking like a nurse: A research-based model of clinical judgment in nursing. J. Nurs. Educ. 45(6): 204-211.

The Health Communication Unit. 2002. Using focus groups. Toronto, ON. Available at: http://www.sswm.info/sites/default/ files/reference_attachments/THCU\%202002\%20Using\%20Focus\% 20Group.pdf 